\title{
Inactivation of the DevS Histidine Kinase of Mycobacterium smegmatis by the Formation of the Intersubunit Disulfide Bond
}

\author{
Jin-Mok Lee ${ }^{1}$, Kwang-Jin Park ${ }^{1}$, Min-Ju Kim ${ }^{1}$, In-Jeong $\mathrm{Ko}^{2}$ and Jeong-II Oh ${ }^{1 *}$ \\ ${ }^{1}$ Department of Microbiology, Pusan National University, Busan 609-735, Korea \\ ${ }^{2}$ Korea Science Academy of KAIST, Busan 614-822, Korea
}

Received June 4, 2010 /Accepted June 16, 2010

\begin{abstract}
The DevSR two-component system is a major regulatory system involved in redox sensing in Mycobacterium smegmatis. The DevSR system consists of the DevS histidine kinase and its cognate DevR response regulator. When exposed to hypoxic conditions, the DevS histidine kinase is activated to phosphorylate the DevR response regulator, leading to the transcriptional activation of the DevR regulation. The ligand-binding state of the heme embedded in the N-terminal GAF domain of DevS determines the kinase activity of DevS. In this study, we demonstrated that the redox-responsive cysteine (C547) in the C-terminal kinase domain is involved in the redox-dependent control of DevS kinase activity. The formation of an intersubunit disulfide bond between the C547 residues in the presence of $\mathrm{O}_{2}$ led to inactivation of DevS kinase activity. The reduction of the oxidized DevS with reductants such as $\beta$-mercaptoethanol and dithiothreitol resulted in the restoration of DevS kinase activity. It was demonstrated in vivo by complementation test that the substitution of C547 to alanine partially impaired the sensory function of DevS in M. smegmatis.
\end{abstract}

Key words : Cysteine, DevSR two-component system, histidine kinase, mycobacteria, redox sensing

\section{Introduction}

Mycobacterium smegmatis is a fast-growing and obligately aerobic mycobacterium. When the growth condition of $M$. smegmatis is transited from aerobic to hypoxic conditions in a gradual way, the genes under the control of the DevSR two-component system are induced such that the bacterium is prepared to enter the nonreplicating, latent state $[5,7,8,12,29,30]$. The DevSR two-component system consists of the DevS histidine kinase (HK) and its cognate DevR response regulator (RR) $[18,20,21]$. The DevS HK is composed of the N-terminal sensory domain with two tandem GAF domains (GAF-A and GAF-B from its N-terminus) and the C-terminal HK domain $[2,19]$. The GAF-A domain serves as a heme-binding domain and comprises a $\beta$-sheet composed of five antiparellel $\beta$-strands and four a-helices [2,19]. A $b$-type heme is positioned in the heme-binding pocket formed between the $\beta$-sheet and the long loop between a2and a3-helices $[2,10,19,23]$. The ligand-binding state at the distal axial position of the DevS heme was demonstrated to control the kinase activity of DevS [14]. The $\mathrm{O}_{2}$-bound (oxyferrous) form of DevS is inactive, whereas the un-

\footnotetext{
*Corresponding author

Tel : +82-51-510-2593, Fax : +82-51-514-1778

E-mail : joh@pusan.ac.kr
}

liganded ferrous (deoxyferrous) as well as the NO-bound form is active [14].

In this paper, we report the evidence that C547 of DevS is involved in inactivation of DevS HK activity in the presence of $\mathrm{O}_{2}$ and discuss the implications of this finding in terms of oxygen-sensory function of DevS.

\section{Materials and Methods}

Bacterial strains, plasmids, and culture conditions The bacterial strains and plasmids used in this study are listed in Table 1. M. smegmatis strains were grown aerobically on Middlebrook 7H9 medium (Difco, Sparks, MD) supplemented with $0.2 \%(\mathrm{w} / \mathrm{v})$ glucose at $37^{\circ} \mathrm{C}$. When required, hygromycin $(50 \mu \mathrm{g} / \mathrm{ml})$ was added to the growth medium. To prevent clumping of bacterial cells, tween 80 was added in $7 \mathrm{H} 9$ medium to a final concentration of $0.02 \%(\mathrm{v} / \mathrm{v}) . M$. smegmatis strains were grown either aerobically in a 250-ml Erlenmeyer flask filled with $100 \mathrm{ml}$ of 7H9-glucose medium on a gyratory shaker (200 rpm) to an optical density at 600 $\mathrm{nm}\left(\mathrm{OD}_{600}\right)$ of 0.5 or under hypoxic conditions in a $250-\mathrm{ml}$ flask filled with $150 \mathrm{ml}$ of 7H9-glucose medium and tightly sealed with a rubber septum on a gyratory shaker (200 rpm) for $15 \mathrm{hr}$ following inoculation of the medium with aerobically grown preculture to $\mathrm{OD}_{600}$ of 0.05 , which allowed a 
Table 1. Bacterial strains and plasmids used in this study

\begin{tabular}{|c|c|c|}
\hline Strain or plasmid & Relevant phenotype or genotype & Source or reference \\
\hline \multicolumn{3}{|l|}{ Strains } \\
\hline \multicolumn{3}{|l|}{ E. coli } \\
\hline DH5a & (\$80dlacZ $\Delta$ M15) $\Delta l a c U 169$ recZ1 endA1 hsdR17 supE44 thi-l gyrA96 relA1 & [11] \\
\hline BL21(DE3) & $\mathrm{F}^{-}$ompT $h s d S_{\mathrm{B}}\left(\mathrm{r}_{\mathrm{B}}^{-} \mathrm{m}_{\mathrm{B}}^{-}\right) d c m$ gal $\lambda(\mathrm{DE} 3)$ & Promega \\
\hline \multicolumn{3}{|l|}{ M. smegmatis } \\
\hline$m c^{2} 155$ & High-transformation-efficiency mutant of M. smegmatis ATCC 607 & [25] \\
\hline$\triangle \operatorname{dev} S$ & devS deletion mutant derived from M. smegmatis $\mathrm{mc}^{2} 155$ & M. J. Kim and J. I. Oh \\
\hline \multicolumn{3}{|c|}{ w } \\
\hline pBSПKS+ & $\mathrm{Ap}^{r} ;$ lacPOZ & Stratagene \\
\hline pNBV1 & $\mathrm{Hyg}^{\mathrm{r}} ; 5.8-\mathrm{kb}$ plasmid derived from p16R1 & [9] \\
\hline pT7-7 & $\begin{array}{l}\text { Ap }{ }^{r} \text {; T7 promoter, RBS, and translation start codon overlapping with } \\
\text { NdeI site }\end{array}$ & [28] \\
\hline pT7SIHis & $\begin{array}{l}\text { pT7-7::1,727-kb NdeI-EcoRI fragment containing devS with } 6 \text { His codons } \\
\text { before its stop codon }\end{array}$ & [14] \\
\hline pT7STHis & $\begin{array}{l}\text { pT7-7::0.739-kb NdeI-EcoRI fragment containing the } 3 \text {-part of } \operatorname{dev} S \text { with } \\
6 \text { His codons before its stop codon }\end{array}$ & [14] \\
\hline pT7SIC422A & pT7SIHis in which the codon for C422 is displaced with GCC & This study \\
\hline pT7SIC547A & pT7SIHis in which the codon for C547 is displaced with GCC & This study \\
\hline pUCSHis & $\begin{array}{l}\text { pUC19::1.715-kb BamHI fragment containing devS with } 6 \text { His codons } \\
\text { before its stop codon; devS is colinear to lacZ }\end{array}$ & M. J. Kim and J. I. Oh \\
\hline pNBV1SHis & pNBV1::1.715-kb BamHI fragment from pUCSHis; devS is colinear to lacZ & M. J. Kim and J. I. Oh \\
\hline pNBV1SH150A & pNBV1SHis in which the codon for H150 is displaced with GCT & This study \\
\hline pNBV1SC422A & pNBV1SHis in which the codon for H422 is displaced with GCC & This study \\
\hline pNBV1SC547A & pNBV1SHis in which the codon for H547 is displaced with GCC & This study \\
\hline
\end{tabular}

gradual depletion of $\mathrm{O}_{2}$ from the growth medium. Escherichia coli strains were grown on Luria-Bertani (LB) medium at $37^{\circ} \mathrm{C}$ or $30^{\circ} \mathrm{C}$

\section{DNA manipulation and electroporation techniques}

Standard protocols or manufacturer's instructions were followed for recombinant DNA manipulations [22]. The introduction of plasmids into M. smegmatis strains was carried out as described elsewhere [25].

\section{Site-directed mutagenesis}

To mutate H150, C422, and C547 of DevS to alanine, site-directed mutagenesis was performed using the plasmid pUCSHis as the template plasmid and the Quick Change Site-Directed Mutagenesis Kit (Stratagene, La Jolla, CA). Synthetic oligonucleotides 33 bases long containing an alanine codon in place of the histidine or cysteine codon in the middle of their sequences were used to mutagenize the histidine or cysteine codon, respectively. Mutation was verified by DNA sequencing. For the construction of plasmids for complementation test, 1.7-kb Bam HI fragments from the mutated pUCSHis plasmids were cloned into pNBV1 with devS being collinear to lacZ, yielding pNBV1SH150A,
pNBV1SC422A, and pNBV1SC547A. For the construction of the overexpression plasmids, pT7SIHis was directly employed as the template for site-directed mutagenesis and the resulting plasmids were pT7SIC422A and pT7SIC547A.

\section{Protein purification}

Intact DevS: The E. coli BL21 (DE3) strain carrying pT7SIHis, pT7SIC422A or pT7SIC547A was grown in LB medium containing $100 \mu \mathrm{g} / \mathrm{ml}$ ampicillin aerobically at $37^{\circ} \mathrm{C}$ to $\mathrm{OD}_{600}$ of $0.4-0.5$. The $\operatorname{dev} S$ gene was induced by the addition of IPTG to a final concentration of $0.5 \mathrm{mM}$, and cells were further grown for $4 \mathrm{hr}$ at $30^{\circ} \mathrm{C}$. After harvesting of 21 culture, cells were resuspended in $30 \mathrm{ml}$ of buffer A [20 $\mathrm{mM}$ Tris- $\mathrm{HCl}$ (pH 8.0) containing $100 \mathrm{mM} \mathrm{NaCl}$ ] and disrupted by two passages through a French pressure cell. Following DNase I treatment (150 units) in the presence of $10 \mathrm{mM} \mathrm{MgCl}_{2}$ for $30 \mathrm{~min}$ on ice, cell-free crude extracts were obtained by centrifugation two times at $20,000 \times \mathrm{g}$ for $20 \mathrm{~min}$. After addition of imidazole to a final concentration of $5 \mathrm{mM}$, $1 \mathrm{ml}$ of $80 \%$ (v/v) Ni-Sepharose High Performance resin (Amersham Biosciences, Piscataway, NJ) was added to the crude extracts and mixed gently by shaking for $2 \mathrm{hr}$ on ice. The protein-resin mixture was loaded into a column, and 
the column was washed with 40 volumes of buffer A containing $10 \mathrm{mM}$ imidazole, followed by 20 volumes of buffer A containing $50 \mathrm{mM}$ imidazole. The His 6 -tagged DevS protein was eluted with buffer A containing $200 \mathrm{mM}$ imidazole. The fractions containing the DevS protein were collected and dialyzed against 21 of $20 \mathrm{mM}$ Tris- $\mathrm{HCl}$ ( $\mathrm{pH}$ 8.0) overnight at $4^{\circ} \mathrm{C}$. The desalted DevS was concentrated by means of ultrafiltration (membrane YM10; Millipore Co., Bedford, MA).

Truncated DevS (C-DevS): C-DevS was overexpressed in an E. coli BL21 (DE3) strain carrying pT7STHis. The strain was grown and the devS gene was induced as described for overexpression of the intact DevS. The purification procedure of C-DevS was the same as that of the intact DevS except for the washing and elution conditions during affinity chromatography: 20 volumes of buffer B [20 mM Tris- $\mathrm{HCl}$ (pH 8.0) containing $500 \mathrm{mM} \mathrm{NaCl}$ ] containing $5 \mathrm{mM}$ imidazole in the first wash step, 40 volumes of buffer B containing $20 \mathrm{mM}$ imidazole in the second wash step, and elution with buffer B containing $250 \mathrm{mM}$ imidazole. The purified C-DevS was concentrated and stored at $-70^{\circ} \mathrm{C}$. When required in the purification procedures of DevS and C-DevS, $\beta$-mercaptoethanol was added to all buffers used in the purification procedure to a final concentration of $20 \mathrm{mM}$.

\section{In vitro autophosphorylation assay}

Protein phosphorylation was performed at either room temperature (RT) or $30^{\circ} \mathrm{C}$ in an assay buffer containing the appropriate protein components, $20 \mathrm{mM}$ Tris- $\mathrm{HCl}$ ( $\mathrm{pH} 8.0$ ), $50 \mathrm{mM} \mathrm{KCl}$, and $5 \mathrm{mM} \mathrm{MgCl}_{2}$. The reaction was initiated by the addition of a mixture of $\left[\mathrm{\gamma}^{32} \mathrm{P}\right] \mathrm{ATP}$ and unlabeled ATP to a final concentration of $200 \mu \mathrm{M}$ $(1,000 \mathrm{Ci} / \mathrm{mol})$. In the case of time course experiments, samples $(10 \mu \mathrm{l})$ were removed at various time intervals, and reactions were stopped by the addition of $3 \mu \mathrm{l}$ of $5 \times$ loading buffer $[50 \mathrm{mM}$ Tris- $\mathrm{HCl},(\mathrm{pH}$ 6.8), 4\% (w/v) SDS, 20\% (w/v) glycerol, $20 \mathrm{mM}$ dithiothreitol (DTT), 1\% (v/v) B-mercaptoethanol, 0.1\% (w/v) bromophenol blue, and $100 \mathrm{mM}$ EDTA]. Samples were denatured for $40 \mathrm{~min}$ at RT in loading buffer, and ${ }^{32} \mathrm{P}$-labeled PrrB was resolved by SDS-PAGE using $12.5 \%(\mathrm{w} / \mathrm{v})$ polyacrylamide gels. Gels were dried at $80^{\circ} \mathrm{C}$ under vacuum, and the labeled proteins were visualized by autoradiography. The dried gel was autoradiographed with an X-ray film (BIOMAX XAR Film: Kodak, Rochester, NY) in an exposure cassette at $-70^{\circ} \mathrm{C}$ for $1-3$ days.

\section{Determination of protein concentration and non- reducing SDS-PAGE}

Protein concentration was determined by the Bio-Rad protein assay kit (Bio-Rad, Hercules, CA) with bovine serum albumin as the standard protein. Non-reducing SDS-PAGE was conducted using the sample buffer without $\beta$-mercaptoethanol and DTT in the same way as SDS-PAGE.

\section{Quantitative real-time PCR (qRT-PCR)}

RNA isolation from M. smegmatis strains was carried out as described elsewhere [17] after disrupting M. smegmatis cells using TRIzol (Invitrogen, Carlsbad, CA) and Fastprep 120 beadbeater. cDNA was synthesized by RT-\&GO ${ }^{\mathrm{TM}}$ Mastermix reverse transcriptase (MPbio, Eschwege, Germany) according to the manufacturer's instruction. Synthesis of cDNA was performed using $1 \mathrm{ng}$ of the isolated total RNA as the template as well as RT-16sr(-) primer (5'-ACAAC GCTCGGACCCTAC-3') and Rihsp(-) primer (5'-CGCCCG TTGGTCTCCTTCTTC- $\left.3^{\prime}\right)$ for the 16S rRNA and $h s p X$ genes, respectively. For qRT-PCR, RT-16sr(+) primer (5'-CTGGGA CTGAGATACGGC-3') and RT-16sr(-) primer for the $16 \mathrm{~S}$ rRNA gene as well as $\operatorname{Rihsp}(+)$ primer $\left(5^{\prime}\right.$ - GGGTCTG CCGTCGTGGGCCTC-3') and Rihsp(-) primer for the $h s p X$ gene were used. PCR reaction was performed using DyNamo SYBR Green qPCR kit (Bio-Rad). qRT-PCR was performed in a $20 \mu \mathrm{l}$ mixture containing $10 \mathrm{ng}$ of the template cDNA, 15 pmol each of the primers, $10 \mu \mathrm{l}$ of $\mathrm{iQ}^{\mathrm{TM}} \mathrm{SYBR}$ Green supermix (Bio-Rad), and $7 \mu \mathrm{l}$ of distilled $\mathrm{H}_{2} \mathrm{O}$. Thermal cycling began with the initial step at $94^{\circ} \mathrm{C}$ for $5 \mathrm{~min}$ and followed by 40 cycles of $94^{\circ} \mathrm{C}$ for $1 \mathrm{~min}, 52^{\circ} \mathrm{C}$ for 30 sec, and $72^{\circ} \mathrm{C}$ for 14 sec. qRT-PCR data were analyzed by MJ Opiconmonitor analysis software version 3.1 (Bio-Rad).

\section{Results and Discussion}

\section{Inactivation of DevS under oxidizing conditions}

The intact DevS and truncated form of DevS (C-DevS) proteins were heterologously overexpressed in E. coli and purified to a homogeneity. When DevS was purified under reducing conditions, i.e., in the presence of $20 \mathrm{mM} \beta$ -mercaptoethanol in all the buffer solutions used during the purification procedure, the purified DevS protein showed autokinase activity (Fig. 1). In contrast, when DevS was purified under oxidizing conditions in the absence of $\beta$ -mercatoethanol, the purified protein did not exhibit autokinase activity. To investigate whether the N-terminal sensory 


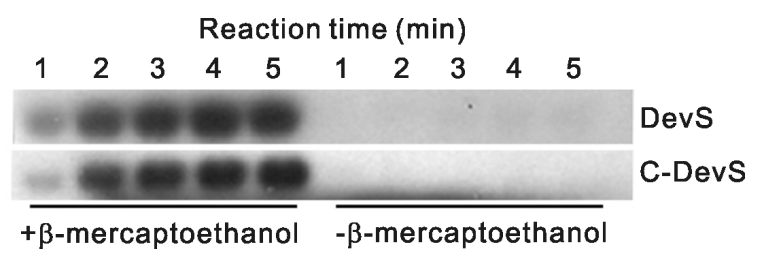

Fig. 1. Autokinase activity of DevS and C-DevS purified in the presence or absence of $\beta$-mercaptoethanol. The proteins were purified using the buffer solutions with $(+)$ or without (-) $20 \mathrm{mM}$ B-mercaptoethanol. The autophosphorylation reactions were performed by using $80 \mathrm{pmol}$ each of purified DevS and C-DevS and $200 \mu \mathrm{M}(1000 \mathrm{Ci} / \mathrm{mol})$ ATP at $30^{\circ} \mathrm{C}$. At the time points indicated, samples (10 ul) were removed and added to $3 \mu$ of sample buffer to stop the reaction. The phosphorylation of the proteins was assayed by SDS-PAGE and subsequent autoradiography.

domain of DevS is responsible for inactivation of DevS under oxidizing conditions, the autophosphorylation assay was performed using C-DevS in which $343 \mathrm{~N}$-terminal amino acids have been removed. As shown in Fig. 1, the C-DevS protein purified in the presence of $\beta$-mercaptoethanol had autokinase activity, where that purified in the absence of $\beta$-mercaptoethanol did not show autokinase activity like the intact DevS. This result indicates that the DevS HK is inactivated in air without $\beta$-mercaptoethanol and that the C-terminal kinase domain of DevS, but not its N-terminal sensory domain containing heme, is involved in inactivation of DevS kinase activity.

We next examined whether the treatment of the oxidized DevS with various reductants led to a restoration of DevS kinase activity. As shown in Fig. 2, the reduction of DevS purified in the absence of $\beta$-mercaptoethaol with either $\beta$ -mercaptoethanol or DTT brought about the restoration of DevS kinase activity, while the treatment of the DevS protein with ascorbate or reduced glutathione did not. As expected, the DevS protein purified in the presence of $\beta$-mercaptoethanol displayed strong autokinase activity. This result strongly suggests that inactivation of DevS kinase activity in air in the absence of any reductants may be due to the formation of (a) disulfide bond(s) between cysteine residues in the C-terminal kinase domain of DevS, since $\beta$-mercaptoethanol and DTT are strong reductants that can reduce a disulfide bond to the thiol groups.

Disulfide bond formation is involved in the inactivation of DevS under oxidizing conditions

We examined the primary sequence of the C-terminal kinase

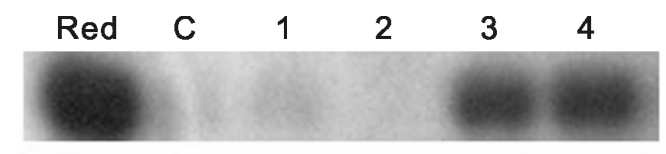

Fig. 2. Effect of several reductants on DevS autokinase activity. The autophosphorylation reactions were performed by using either reduced or oxidized DevS $(80 \mathrm{pmol})$ and $200 \mu \mathrm{M}(1000 \mathrm{Ci} / \mathrm{mol}) \mathrm{ATP}$ at $30^{\circ} \mathrm{C}$ for $5 \mathrm{~min}$. The oxidized DevS was incubated with $10 \mathrm{mM}$ sodium ascorbate (lane 1), $10 \mathrm{mM}$ glutathione (lane 2), $10 \mathrm{mM} \beta$ -mercaptoethanol (lane 3), or $10 \mathrm{mM}$ DTT (lane 4) at RT for $30 \mathrm{~min}$ before the addition of ATP to start autophosphorylation reaction. As the controls, reduced DevS (Red) purified in the presence of $\beta$-mercaptoethanol and oxidized DevS (C) without the treatment of any reductants were included in the experiment. After autophosphorylation reactions, samples $(10 \mu \mathrm{l})$ were added to 3 $\mu l$ of sample buffer to stop the reaction. The phosphorylation of the proteins was assayed by SDS-PAGE and subsequent autoradiography.

domain of DevS corresponding to C-DevS and found that two cysteines residues (C422 and C547) occur in the C-terminal kinase domain of DevS. In order to assess whether these cysteine residues are involved in the formation of the disulfide bond, we replaced C422 and C547 with alanine by site directed mutagenesis and subjected the mutant forms of DevS (C422A DevS and C547A DevS) purified under oxidizing conditions to non-reducing SDS-PAGE (Fig. 3). As the controls, the wild-type (WT) DevS and C-DevS were also

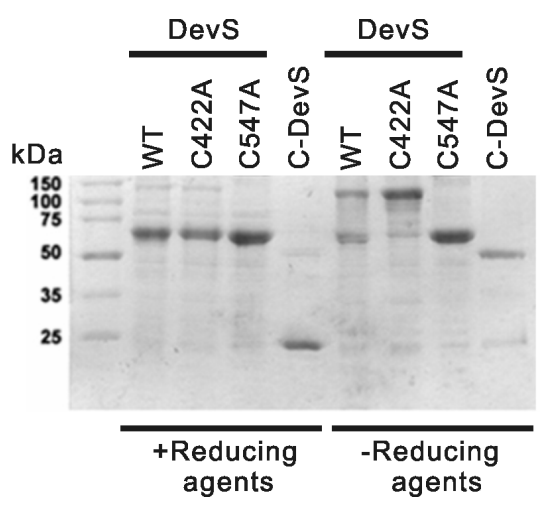

Fig. 3. Non-reducing SDS-PAGE of the C422A and C547A mutant forms of DevS purified in the absence of $\beta$ -mercaptoethanol. The purified proteins $(80 \mathrm{pmol})$ were resolved by non-reducing SDS-PAGE $(12.5 \%$ polyacrylamide gel) and visualized by staining with Coomassie brilliant blue. +Reducing agents: DTT and $\beta$ mercaptoethanol were included in sample buffer, -Reducing agents: no DTT and $\beta$-mercaptoethanol in sample buffer, WT: wild-type form DevS, C-DevS: truncated DevS lacking the N-terminal sensory domain. 
subjected to non-reducing SDS-PAGE. When the reducing agents such as $\beta$-mercaptoethanol and DTT were not included in the sample buffer of SDS-PAGE, the protein band of $130 \mathrm{kDa}$ was observed for WT DevS. However, when the sample buffer containing the reducing agents was used, the band of $130 \mathrm{kDa}$ disappeared and the major band of $65 \mathrm{kDa}$, which corresponds to the molecular mass of the monomeric DevS, was observed for WT DevS. In the case of C-DevS, the homodimeric protein $(46 \mathrm{kDa})$ and monomeric protein (23 kDa) were also observed in the gel in the absence and presence of the reducing agents in the sample buffer, respectively. This result indicates that WT DevS and C-DevS purified under oxidizing conditions in the absence of $\beta$ -mercaptoethanol are dimerized and that the disulfide-reducible reductants such as $\beta$-mercaptoethanol and DTT can disrupt the intermolecular dimerization of DevS and C-DevS. The C422A mutant form of DevS showed the same band pattern as WT DevS, whereas the C547A mutant form of DevS showed the band of $65 \mathrm{kDa}$, even though the sample buffer without the reducing agents was employed, indicating that the C547 residue of DevS is responsible for intermolecular dimerization through disulfide bond formation.

To examine the effect of C422A and C547A mutations on the kinase activity of DevS, we purified the WT and mutant forms of DevS in the presence or absence of $\beta$-mercaptoethanol and determined their autokinase activities. As shown in Fig. 4, the autokinase activities of both WT DevS and C422A DevS were inactivated when the proteins were purified in the absence of $\beta$-mercaptoethanol. In contrast, C547A DevS purified in the absence of $\beta$-mercaptoethanol showed the similar levels of autokinase activity to that purified in the

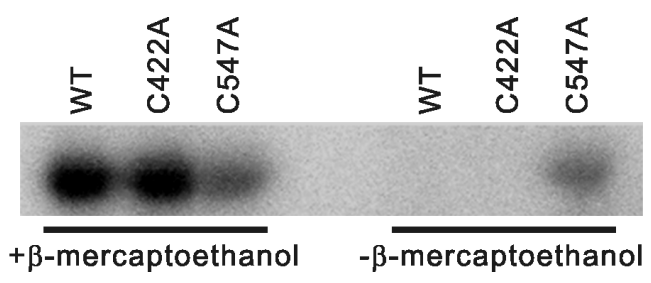

Fig. 4. Autophosphoryltion assay of the C422A and C547A mutant forms of DevS. The autophosphorylation reactions were performed by using 80 pmol each of wild-type (WT) DevS, C422A DevS, and C547A DevS purified in the presence $(+)$ or absence $(-)$ of $\beta$-mercaptoethanol and $200 \mu \mathrm{M}(1000 \mathrm{Ci} / \mathrm{mol})$ ATP at $30^{\circ} \mathrm{C}$ for $3 \mathrm{~min}$. After phosphorylation reactions, samples were added to $3 \mu \mathrm{l}$ of sample buffer to stop the reaction. The phosphorylation of the proteins was assayed by SDS-PAGE and subsequent autoradiography. presence of $\beta$-mercaptoethanol. It is noteworthy that the C547A mutation caused a decrease in DevS autokinase activity, compared with WT DevS and C422A DevS. This result strongly suggests that the dimerization of DevS by the intermolecular formation of the disulfide bond results in inactivation of DevS kinase activity and that C547A mutation compromises DevS kinase activity.

To assess the functionality of the mutant forms of DevS in vivo, complementation analysis using a devS deletion mutant $(\Delta d e v S)$ of $M$. smegmatis was performed. The reporter gene used in the complementation test was the $h s p X$ gene whose expression was known to be strongly induced under hypoxic conditions by the DevSR two-component system $[1,3,4,6,16,21,24,29,31]$. The mutated genes encoding C422A and C547A DevS were cloned into the expression shuttle vector pNBV1 and introduced into the $\Delta \operatorname{dev} S$ mutant of $M$. smegmatis. The H150A mutant form of DevS was also included in this experiment. The H150 residue of DevS serves as the distal axial ligand of the heme iron and the point mutation of H150A was reported to lead to the loss of heme [14]. We reasoned that the corresponding amino acid might be important in the sensory function of DevS if a mutant form of DevS does not complement the $\Delta$ devS mutant of $M$. smegmatis in terms of hypoxic induction of $h s p X$. The $\Delta d e v S$ mutant strain of $M$. smegmatis with the empty pNBV1 vector was used as a negative control. As expected, expression of $h s p X$ in the $\Delta d e v S$ mutant strain with pNBV1 was not induced under hypoxic conditions, whereas the introduction of devS into the mutant led to the restoration of hypoxic induction of $h s p X$ to the level observed in the WT strain of M. smegmatis with pNBV1 (Fig. 5). The introduction of H150A DevS into the $\Delta$ devS mutant strain did not lead to the hypoxic induction of $h s p X$, indicating that the mutant form of DevS lost the sensory function due to the lack of the heme responsible for $\mathrm{O}_{2}$ sensing. The C422A mutant form of DevS induced $h s p X$ expression under hypoxic conditions to the level observed in the the $\Delta d e v S$ mutant with WT DevS, indicating that the $\mathrm{O}_{2}$-sensory function of DevS is not impaired by C422A mutation. Although the C547A DevS induced $h s p X$ expression by 11 fold under hypoxic conditions relative to under aerobic conditions, the induction fold was significantly smaller than that exerted by WT DevS (43.9 fold) and C422A DevS (40.9 fold), implying that the C547A mutation brings about the partial loss of the $\mathrm{O}_{2}$-sensory function of DevS. The 11-fold hypoxic induction of $h s p X$ observed in the $\Delta d e v S$ mutant with C547A DevS is 


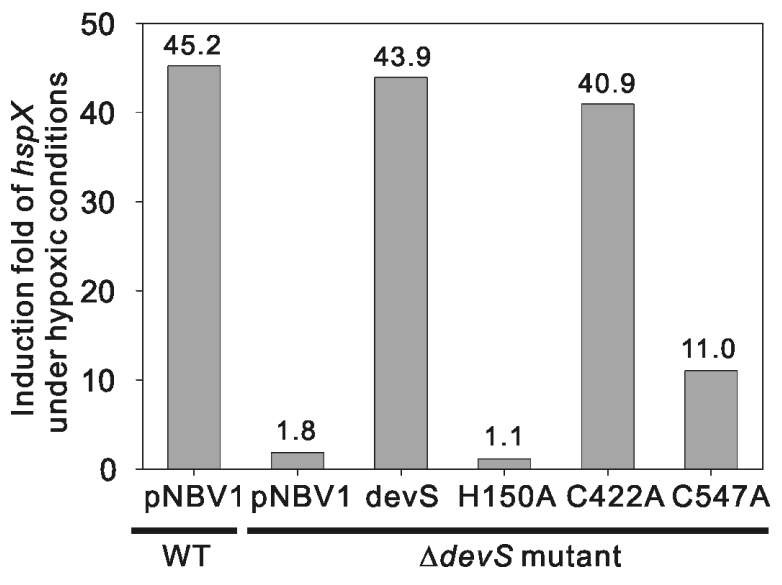

Fig. 5. Effect of H150A, C422A, and C547A mutations on the sensory function of DevS in vivo. Complementation test was performed by means of qRT-PCR. The $h s p X$ gene whose expression is known to be induced strongly under hypoxic conditions by the DevSR two-component system was used as a reporter gene. The DevS mutant strain of M. smegmatis was complemented by the plasmids pNBV1SHis (devS), pNBV1SH150A (H150A), pNBV1SC422A (C422A), and pNBV1SC547A (C547A). The strains were grown under either aerobic or hypoxic conditions. The relative levels of $h s p X$ mRNA were determined from the threshold values that were normalized to $16 \mathrm{~S}$ rRNA gene expression. Induction fold of $h s p X$ indicates the level of $h s p X$ mRNA of hypoxic culture relative that of aerobic culture.

likely due to $\mathrm{O}_{2}$ sensing by heme in its N-terminal sensory domain.

\section{Model for $\mathrm{O}_{2}$ sensing by DevS HK}

On the basis of the results presented here as well as those reported previously, we present a model for $\mathrm{O}_{2}$ sensing by DevS HK. The N-terminal domain of DevS contains two tandem GAF domains (GAF-A and GAF-B) [19,23]. The GAF-A domain serves as the heme-binding domain [2,19]. The ligand-binding state of the heme in DevS determines its kinase activity $[13,14,26]$. The heme in DevS is in a deoxy-ferrous form under oxygen-limiting (hypoxic) and anaerobic conditions and is converted to an oxy-ferrous form under aerobic conditions $[13,14,26]$. The DevS HK with the deoxy-ferrous heme possesses the active kinase activity, whereas that with the oxy-ferrous heme does not $[13,14,26]$. Therefore, when M. smegmatis is transitted from aerobic conditions to hypoxic conditions, the DevR RR is phosphorylated at the conserved aspartate (D63) residue in its N-terminal receiver domain and activated to serve as an active tran- scriptional regulator for the DevR regulon whose expression primes the bacterium to survive in a nonreplicating, latent state under hypoxic or anaerobic conditions. In the case of DevS of M. smegmatis, thiol-disulfide conversion at C547 also appears to be involved in redox sensing of DevS. Oligomerization of DevS polypeptides by the formation of the disulfide bond inactivates the DevS kinase activity under oxidizing conditions. Gel filtration chromatography revealed that the active DevS protein purified in the presence of $\beta$ -mercaptoethanol exists as a homodimer, whereas the oxidized, inactive DevS protein is in the form of homotetramer and more-complex oligomer (data not shown). The control of $\mathrm{HK}$ activity by the thiol-disulfide exchange at redox-active cysteines has been demonstrated for the RegB HK of Rhodobacter capsulatus and the ArcB HK of E. coli $[15,27]$. In both the cases, the formation of the disulfide bond leads to inactivation of autokinase activities of the proteins. Exposure of RegB to oxidizing conditions results in the formation of an intermolecular disulfide bond at $\mathrm{C} 265$, which is similar to the case of DevS [27]. ArcB was also shown to be inactivated by the formation of intermolecular disulfide bonds [15]. The conversion of the thiol groups of C180 and C241 to the disulfides is caused not directly by $\mathrm{O}_{2}$ but by oxidized quinones in the respiratory electron transport chain [15].

It seems likely that the major $\mathrm{O}_{2}$-sensing function of DevS is conducted by the heme embedded in its GAF-A domain, since the residue corresponding to C547 of DevS is not conserved in other DevS homologs including DosS and DosT of Mycobacterium tuberculosis.

\section{Acknowledgment}

This work was supported by the Korean Research Foundation Grant funded by Korean Government (MOEHRD, Basic Research Promotion Fund) (KRF-2007- 521-C00251).

\section{References}

1. Boon, C., R. Li, R. Qi, and T. Dick. 2001. Proteins of Mycobacterium bovis BCG induced in the Wayne dormancy model. J. Bacteriol. 183, 2672-2676.

2. Cho, H. Y., H. J. Cho, Y. M. Kim, J. I. Oh, and B. S. Kang. 2009. Structural insight into the heme-based redox sensing by DosS from Mycobacterium tuberculosis. J. Biol. Chem. 284, 13057-13067.

3. Cunningham, A. F. and C. L. Spreadbury. 1998. Mycobacterial stationary phase induced by low oxygen tension: Cell wall 
thickening and localization of the 16-kilodalton alpha-crystallin homology. J. Bacteriol. 180, 801-808.

4. Desjardin, L. E., L. G. Hayes, C. D. Sohaskey, L. G. Wayne, and K. D. Eisenach. 2001. Microaerophilic induction of the alpha-crystallin chaperone protein homologue ( $h s p X)$ mRNA of Mycobacterium tuberculosis. J. Bacteriol. 183, 5311-5316.

5. Dick, T., B. H. Lee, and B. Murugasu-Oei. 1998. Oxygen depletion induced dormancy in Mycobacterium smegmatis. FEMS Microbiol. Lett. 163, 159-164.

6. Florczyk, M. A., L. A. McCue, R. F. Stack, C. R. Hauer, and K. A. McDonough. 2001. Identification and characterization of mycobacterial proteins differentially expressed understanding and shaking culture conditions, including Rv2623 from a novel class of putative ATP-binding proteins. Infect. Immun. 69, 5777-5785.

7. Gill, W. P., N. S. Harik, M. R. Whiddon, R. P. Liao, J. E. Mittler, and D. R. Sherman. 2009. A replication clock for Mycobacterium tuberculosis. Nat. Med. 15, 211-214.

8. Honaker, R. W., R. L. Leistikow, I. L. Bartek, and M. I. Voskuil. 2009. Unique roles of DosT and DosS in DosR regulon induction and Mycobacterium tuberculosis dormancy. Infect. Immun. 77, 3258-3263.

9. Howard, N. S., J. E. Gomez, C. Ko, and W. R. Bishai. 1995. Color selection with a hygromycin-resistance-based Escherichia coli-mycobacterial shuttle vector. Gene 166, 181-182.

10. Ioanoviciu, A., E. T. Yukl, P. Moenne-Loccoz, and P. R. O. de Montellano. 2007. DevS, a heme-containing two-component oxygen sensor of Mycobacterium tuberculosis. Biochemistry 46, 4250-4260.

11. Jessee, J. 1986. New subcloning efficiency competent cells: $>1$ $\mathrm{X} 10^{6}$ transformants $/ \mu \mathrm{g}$. Focus $8,9$.

12. Kendall, S. L., F. Movahedzadeh, S. C. G. Rison, L. Wernisch, T. Parish, K. Duncan, J. C. Betts, and N. G. Stoker. 2004. The Mycobacterium tuberculosis dosRS two-component system is induced by multiple stresses. Tuberculosis $\mathbf{8 4}$, 247-255.

13. Kumar, A., J. C. Toledo, R. P. Patel, J. R. Lancaster, and A. J. C. Steyn. 2007. Mycobacterium tuberculosis DosS is a redox sensor and DosT is a hypoxia sensor. Proc. Natl. Acad. Sci. USA 104, 11568-11573.

14. Lee, J. M., H. Y. Cho, H. J. Cho, I. J. Ko, S. W. Park, H. S. Baik, J. H. Oh, C. Y. Eom, Y. M. Kim, B. S. Kang, and J. I. Oh. 2008. $\mathrm{O}_{2}$ - and NO-sensing mechanism through the DevSR two-component system in Mycobacterium smegmatis. J. Bacteriol. 190, 6795-6804.

15. Malpica, R., B. Franco, C. Rodriguez, O. Kwon, and D. Georgellis. 2004. Identification of a quinone-sensitive redox switch in the ArcB sensor kinase. Proc. Natl. Acad. Sci. USA 101, 13318-13323.

16. Muttucumaru, D. G. N., G. Roberts, J. Hinds, R. A. Stabler, and T. Parish. 2004. Gene expression profile of Mycobacterium tuberculosis in a non-replicating state. Tuberculosis 84, 239-246.

17. Oelmuller, U., N. Kruger, A. Steinbuchel, and C. G. Friedrich. 1990. Isolation of procaryotic RNA and detection of specific mRNA with biotinylated probes. J. Microbiol.
Methods 11, 12.

18. Park, H. D., K. M. Guinn, M. I. Harrell, R. Liao, M. I. Voskuil, M. Tompa, G. K. Schoolnik, and D. R. Sherman. 2003. Rv3133c/dosR is a transcription factor that mediates the hypoxic response of Mycobacterium tuberculosis. Mol. Microbiol. 48, 833-843.

19. Podust, L. M., A. Ioanoviciu, and P. R. O. de Montellano. 2008. 2.3 angstrom X-ray structure of the heme-bound GAF domain of sensory histidine. Biochemistry 47, 12523-12531.

20. Roberts, D. M., R. L. P. Liao, G. Wisedchaisri, W. G. J. Hol, and D. R. Sherman. 2004. Two sensor kinases contribute to the hypoxic response of Mycobacterium tuberculosis. J. Biol. Chem. 279, 23082-23087.

21. Saini, D. K., V. Malhotra, D. Dey, N. Pant, T. K. Das, and J. S. Tyagi. 2004. DevR-DevS is a bona fide two-component system of Mycobacterium tuberculosis that is hypoxia-responsive in the absence of the DNA-binding domain of DevR. Microbiology 150, 865-875.

22. Sambrook, J., E. F. Fritsch, and T. Maniatis. 2001. Molecular cloning: a laboratory manual. Cold Spring Harbor Laboratory Press, Cold Spring Harbor, NY.

23. Sardiwal, S., S. L. Kendall, F. Movahedzadeh, S. C. G. Rison, N. G. Stoker, and S. Djordjevic. 2005. A GAF domain in the hypoxia/NO-inducible Mycobacterium tuberculosis DosS protein binds haem. J. Mol. Biol. 353, 929-936.

24. Sherman, D. R., M. Voskuil, D. Schnappinger, R. L. Liao, M. I. Harrell, and G. K. Schoolnik. 2001. Regulation of the Mycobacterium tuberculosis hypoxic response gene encoding alpha-crystallin. Proc. Natl. Acad. Sci. USA 98, 7534-7539.

25. Snapper, S. B., R. E. Melton, S. Mustafa, T. Kieser, and W. R. Jacobs. 1990. Isolation and characterization of efficient plasmid transformation mutants of Mycobacterium smegmatis. Mol. Microbiol. 4, 1911-1919.

26. Sousa, E. H. S., J. R. Tuckerman, G. Gonzalez, and M. A. Gilles-Gonzalez. 2007. DosT and DevS are oxygen-switched kinases in Mycobacterium tuberculosis. Protein Sci. 16, 1708-1719.

27. Swem, L. R., B. J. Kraft, D. L. Swem, A. T. Setterdahl, S. Masuda, D. B. Knaff, J. M. Zaleski, and C. E. Bauer. 2003. Signal transduction by the global regulator RegB is mediated by a redox-active cysteine. EMBO J. 22, 4699-4708.

28. Tabor, S. and C. C. Richardson. 1985. A bacteriophage T7 RNA polymerase/ promoter system for controlled exclusive expression of specific genes. Proc. Natl. Acad. Sci. USA 82, 1074-1078.

29. Voskuil, M. I., K. C. Visconti, and G. K. Schoolnik. 2004. Mycobacterium tuberculosis gene expression during adaptation to stationary phase and low-oxygen dormancy. Tuberculosis 84, 218-227.

30. Wayne, L. G. and C. D. Sohaskey. 2001. Nonreplicating persistence of Mycobacterium tuberculosis. Annu. Rev. Microbiol. $55,139-163$.

31. Yuan, Y., D. D. Crane, R. M. Simpson, Y. Q. Zhu, M. J. Hickey, D. R. Sherman, and C. E. Barry. 1998. The 16-kDa alpha-crystallin (Acr) protein of Mycobacterium tuberculosis is required for growth in macrophages. Proc. Natl. Acad. Sci. USA 95, 9578-9583. 


\title{
초록 : Subunit 간의 disulfide 결합 형성에 의한 Mycobacterium smegmatis DevS histidine kinase의 불활성화
}

\author{
이진목 ${ }^{1} \cdot$ 박광진 $^{1} \cdot$ 김민주 $^{1} \cdot$ 고인정 $^{2} \cdot$ 오정일 $^{1 *}$ \\ ( ${ }^{1}$ 부산대학교 미생물학과, ${ }^{2} \mathrm{KAIST}$ 부설 한국영재학교)
}

DevSR two-component system은 Mycobacterium smegmatis의 redox sensing에 관련된 주요한 regulatory system이다. DevSR system은 DevS histidine kinase와 DevR response regulator로 구성되어 있다. 저산소 조건에서 DevS histidine kinase는 활성화되어 DevR response regulator를 인산화 시키고, 인산화된 DevR response regulator는 DevR regulon의 transcriptional activator로 작용한다. DevS의 kinase activity는 DevS의 N-terminal에 위 치한 GAF domain에 존재하는 heme의 ligand-binding state에 의해 결정된다. 본 연구에서는 C-terminal kinase domain의 redox-responsive cysteine (C547)이 DevS kinase activity의 redox-dependent control과 연관이 있음을 밝혔다. 산소가 존재할 때, C547 residue 사이의 disulfide bond의 형성은 DevS kinase activity를 불활성화 시킨 다. $\beta$-mercaptoethanol과 dithiothreitol과 같은 환원제를 이용하여 산화된 DevS를 환원시켰을 때, DevS kinase activity가 복원된 것이 관찰되었다. 또한, C547을 alanine으로 치환했을 때, M. smegmatis의 DevS의 sensory 기능 을 부분적으로 손상되는 것이 complementation 실험을 통해 in vivo 상에서 증명되었다. 